\title{
A technique to improve the empirical mode decomposition in the Hilbert-Huang transform
}

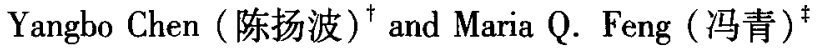 \\ Civil \& Environmental Engineering, University of California, Irvine, CA 92697, USA
}

\begin{abstract}
The Hilbert-based time-frequency analysis has promising capacity to reveal the time-variant behaviors of a system. To admit well-behaved Hilbert transforms, component decomposition of signals must be performed beforehand. This was first systematically implemented by the empirical mode decomposition (EMD) in the Hilbert-Huang transform, which can provide a time-frequency representation of the signals. The EMD, however, has limitations in distinguishing different components in narrowband signals commonly found in free-decay vibration signals. In this study, a technique for decomposing components in narrowband signals based on waves' beating phenomena is proposed to improve the EMD, in which the time scale structure of the signal is unveiled by the Hilbert transform as a result of wave beating, the order of component extraction is reversed from that in the EMD and the end effect is confined. The proposed technique is verified by performing the component decomposition of a simulated signal and a free decay signal actually measured in an instrumented bridge structure. In addition, the adaptability of the technique to time-variant dynamic systems is demonstrated with a simulated time-variant MDOF system.
\end{abstract}

Keywords : time-frequency analysis; Hilbert-Huang transform; component decomposition

\section{Introduction}

The classical system analysis theory and practice have been overwhelmingly dominated by Fourier transform, which represents system characteristics in the frequency domain. However, by computing the global characteristics of the system averaged over the whole period of time, Fourier transform loses the time track of the sudden events and the gradual variation of the system. In reality, many of the signals observed are of transient nature; more importantly, there are many applications that concern the time-variant properties of the system, such as structural health monitoring. In some cases, one can assume a piecewise linear time-invariant system and apply the Fourier transform within each of the limited time windows, which is the short time Fourier transform or the spectrogram analysis (Gröchenig, 2001; Mallat, 1999). However, to represent the time-variant properties of the system, the time width of the window is preferably short, but a short-windowed Fourier transform may not

Correspondence to: Yangbo Chen, Civil \& Environmental Engineering, University of California, Irvine, CA92697, USA.

Tel: 949-824-6359; Fax : 949-824-2117

E-mail: Yangboc@uci.edu

${ }^{\dagger}$ Ph. D Student; ${ }^{\ddagger}$ Professor

Received date: 2003-03-17; Accepted date: 2003-06-02 provide satisfactory resolution to reveal the changes in the system's frequencies. The tradeoff is harder to reach when the phenomenon contains multiple time scales (Huang et al. , 1998).

Take the structural health monitoring application as a particular example. The time-variant properties of the monitored structural system can be attributed to (1) the non-linearity of the system, such as the structural damages due to extreme loading conditions or the structural degradation due to aging or fatigue; (2) non-stationary external excitation, for example the traffic on the bridges and the earthquakes; (3) the time varying boundary conditions and temperature stress affected by the environmental temperature changes; or even (4) the ill-defined system itself, for example, for bridge monitoring based on traffic-induced vibrations, the decrease of the system's natural frequency due to the additional mass of vehicles running on the bridge is oftentimes mistakenly interpreted as a decrease in the stiffness of the bridge structure. These time-variant phenomena have different time scales. For example, the structural degradation may have a time scale of years, while the temperature changes may have a time scale of seasons or days, the nonstationary external excitation a scale of hours or minutes and the structural damage a scale of several seconds.

Therefore, an adaptive alternative for analyzing 
the time-variant system is desirable. One of the promising alternatives is the Hilbert-Huang transform, a time-frequency domain method based on the Hilbert transform proposed by Huang and demonstrated to possess the capacity for analyzing the non-linear nonstationary data by applying it to the numerical results of some classical nonlinear equation systems and data from natural phenomena ( Huang et al. , 1998; 1999). The key part of Huang's method is the empirical mode decomposition (EMD) to decompose the signal into a finite number of components admitting well-behaved Hilbert transforms. The Hilbert-Huang transform has found application in different fields, including earthquake engineering (Zhang and Ma, 2000), wind engineering ( Pan et al., 2003) and structural dynamics (Yang and Lei, 2000; Salvino, 2000).

However, the EMD method has its limitation in distinguishing different components in narrowband signals. In some cases, a narrowband signal is practically considered as only one component; but there are other cases where further decomposition is necessary to reveal its component structure, especially for the purpose of analyzing the system properties based on the free decay response signals. A narrowband signal may contain either (1) components that have adjacent frequencies, or (2) components that may not have adjacent frequencies, but one of them has dominant energy intensity much higher than the other components. In Huang et al. (1998), the EMD method has been used to analyze narrowband signals of the first category, but extremely stringent criteria have to be enforced on the EMD so that it can be carried out to an extreme extent ( 3000 iterations, as in Huang et al. , 1998 ) to distinguish different components, which involves significant effort. Narrowband signals of the second category, commonly found in the structural vibration measurements, have not yet been addressed. They are hardly distinguishable with the EMD method either, as will be shown later with examples in section 5 .

In this study, a technique to improve the EMD method for efficiently distinguishing components in narrowband signals is proposed. The technique is based on the beating phenomenon of waves. Combining with Hilbert transform, the technique possesses better power to reveal the time-variant properties of the system embedded in the measured narrowband signals. The new technique is demonstrated by applying it to the simulated data and the data recorded in the real bridge structure under traffic loading. Prior to the presentation of the proposed technique, some concepts important in time-frequency analysis and an important property of Hilbert transform are reviewed, and the idea of component decomposition contributed by Huang is also reviewed, which is retained and implemented in the proposed technique.

\section{Instantaneous frequency/amplitude}

The cornerstone of time-frequency domain analysis is the concepts of instantaneous frequency and instantaneous amplitude of a signal. The well-known Heisenberg uncertainty theorem (Gröchenig, 2001) has ruled out the possibility of an exact definition of instantaneous frequency based on the Fourier definition of frequency; that is, if the energy is well localized at some special point in time, it is not possible to be localized at any specific value in frequency, and vice versa. Nevertheless, by introducing the Hilbert transform and the notation of the analytic signal, the unique and physical definitions of the instantaneous frequency and the instantaneous amplitude of a signal can be obtained, but with a different physical explanation of frequency, generalized from the conventional Fourier definition.

An arbitrary real signal $S(t)$ has its Hilbert transform $Y(t)$ and its analytic signal $Z(t)$, as

$$
\begin{gathered}
Y(t)=\frac{1}{\pi} \int_{-\infty}^{\infty} \frac{S(\tau)}{t-\tau} \mathrm{d} \tau \\
Z(t)=S(t)+\mathrm{i} Y(t)=a(t) \mathrm{e}^{\mathrm{i} \theta(t)} \\
a(t)=\left[S^{2}(t)+Y^{2}(t)\right]^{1 / 2} \\
\theta(t)=\arctan \frac{Y(t)}{S(t)} \\
\omega(t)=\theta^{\prime}(t)
\end{gathered}
$$

where, $\omega(t)$ is denominated as the instantaneous frequency and $a(t)$ the instantaneous amplitude. The physical explanation (Hahn, 1996) can be achieved in polar coordinate representation of the analytic signal $Z(t)$, in which the instantaneous amplitude $a(t)$ is the length of the phasor and the instantaneous frequency $\omega(t)$ is the instantaneous angular speed of the phasor rotation.

As can be seen from Eq. (1), the local property of $S(t)$ at time $t$ is magnified by Hilbert transform when $\tau$ approaches $t$; indeed, Hilbert transform of a signal is its best local fit of an amplitude and phase varying trigonometric function (Huang et al., 1998).

To show the relationship between the Hilbertbased instantaneous frequency/amplitude in Eqs. (5) and (3) and the conventional Fourier-based frequency/ amplitude, it is worthwhile to revisit an important property of Hilbert transform. 


$$
H[f(t) \mathrm{g}(t)]=f(t) H[\mathrm{~g}(t)]
$$

provided that $f(t)$ and $g(t)$ are signals of non-overlapping spectra and that $f(t)$ has low-pass spectrum while $g(t)$ has high-pass spectrum. $H[S(t)]$ denotes the Hilbert transform of signal $S(t)$. The proof of this property can be found in Hahn (1996).

By inserting a modulated signal $S(t)=A(t) \cos (\omega t)$ into Eqs. (1) through (5), where $A(t)$ is a gradually variant function varying not as fast as $\cos (\omega t)$, and noticing that by Eq. (6) the Hilbert transform of this signal is $Y(t)=A(t) \sin (\omega t)$, the instantaneous amplitude becomes $A(t)$ and the instantaneous frequency becomes $\omega$. Therefore, the Hilbert-based definitions are generalized from the Fourier notations and their physical explanations agree with the authors' intuition.

The Hilbert transform plays a key role in the definitions above. The representation of a signal $S(t)$ in the form of $S(t)=a(t) \cos [\theta(t)]$ is, unfortunately, not unique, which implies that there exist many possible choices of $a(t)$ and $\theta(t)$. The Hilbert transform nevertheless yields a unique pair of $a(t)$ and $\theta(t)$ for $S(t)$ via its analytic signal $Z(t)$ (Hahn, 1996).

The property in Eq. (6) can also help to explain the reason why Hilbert-based analysis can be applied to time-variant system analysis. Given a time variant vibration system as in Eq. (7)

$$
\ddot{x}+2 h_{0}(t) \dot{x}+\omega_{0}^{2}(t) x=f
$$

if the system properties $h_{0}(t)$ and $\omega_{0}(t)$ are gradually varying, by taking Hilbert transform on both sides,

$$
H[\ddot{x}]+2 h_{0}(t) H[\dot{x}]+\omega_{0}^{2}(t) H[x]=H[f]
$$

the time-variant properties of the system are separated from the Hilbert transforms of the excitation and the response signals (compared to the Fourier case where the changing of the system properties will be involved in the Fourier transform ). Indeed, theory to identify time-variant $h_{0}(t)$ and $\omega_{0}(t)$ based on Hilbert transform for a SDOF vibration system has been developed (Feldman, 1994a; 1994b). For a non-linear system, $h_{0}$ and $\omega_{0}$ depend on $x(t)$, which in turn is a time function, non-linear systems can therefore also be represented by Eq. (7).

The Hilbert-based instantaneous frequency/amplitude have rendered the Hilbert-Huang transform the capacity to localize the energy distribution on both time and frequency axes, which, therefore, possessed the power to analyze the time-variant properties of the system.

\section{EMD and Hilbert-Huang transform}

Only a mono-component signal admits a well-behaved Hilbert transform (Huang et al. , 1998). This can be intuitively demonstrated by a signal with two components $S(t)=a_{1}(t) \cos \left(\omega_{1} t\right)+a_{2}(t) \cos \left(\omega_{2} t\right)$. After the Hilbert transform, the analytic signal of $S(t), Z(t)$ gives only one pair of $\omega(t)$ and $a(t)$ at time $t$, they fail to provide frequency/amplitude information of either of the two components. The revolutionary idea of the Hilbert-Huang transform is to decompose a complex signal of multi-components into a finite number of mono-component signals before computing their time-frequency distribution ( Huang et al. , 1998; 1999 ). The process proposed by Huang is referred to as the EMD, which will be reviewed, with its limited capacity shown by examining a simulated narrowband signal.

The method of EMD is developed based on the following observations of the behaviors of a mono-component signal: (1) the number of local extrema and the number of zero crossings must either equal or differ at most by one; and (2) at any point, the mean value of the envelope defined by the local maxima and the envelope defined by the local minima is zero. Any signal satisfying these two conditions is called an intrinsic mode function ( IMF),$F_{\text {IM }}$, which is practically considered as a mono-component signal. The EMD process is therefore to decompose an arbitrary real signal into its IMFs by an operation called sifting. A sifting consists three steps: (1) find out all the local maxima of the signal $S(t)$ and determine the upper envelope $U(t)$, by cubic-spline interpolation of the local maxima; (2) determine the lower envelope $L(t)$ similarly but using the local minima instead; and (3) obtain an IMF by subtracting from the signal the mean of the upper and lower envelopes $F_{\text {IM }}(t)=$ $S(t)-[U(t)+L(t)] / 2$; the remain signal $R(t)=$ $S(t)-F_{\mathrm{IM}}(t)$ is referred to as the residual. The first IMF is resulted from the first sifting, and then the residual $R(t)$ is treated as the signal in the second sifting to obtain the second IMF. The sifting operation is thus repeated until all the IMFs are extracted and the residual becomes a monotonic trend. The advantage of the EMD is that it decomposes the signal according to the intrinsic time scales defined by the local extrema at each round of sifting; therefore, it is an adaptive process able to deal with time-variant signal with complex time scale structures.

However, the EMD has limitations in distinguishing components in narrowband signals. The first reason is the end effect issue. The EMD relies on cubicspline interpolation to determine the upper and lower envelopes of the signal, but the absence of the control 
knots and constrain conditions at both ends has made the interpolation around both ends highly unreliable. The end effect can further swing inwards and distort the signal, which is worse for IMFs with a longer time scale. The second reason is the order of the IMF extractions. In the EMD process, an IMF with a shorter time scale (hence a higher frequency) is extracted before those with lower frequencies. Therefore, errors are accumulated towards the lower modes that may be of more importance for system analysis because they usually carry more energy and are relatively better measured with less noise. But the accumulated error plus the end effect may have deformed the low modes. And the third reason is EMD's unsatisfactory resolution. It has to be carried out to extreme extent to distinguish components in narrowband signals of first category (Huang et al. , 1998). For narrowband signals of the second category, especially when a higher mode with less energy is riding on a lower mode carrying much more energy, the humps of the higher mode may not properly show up as the local

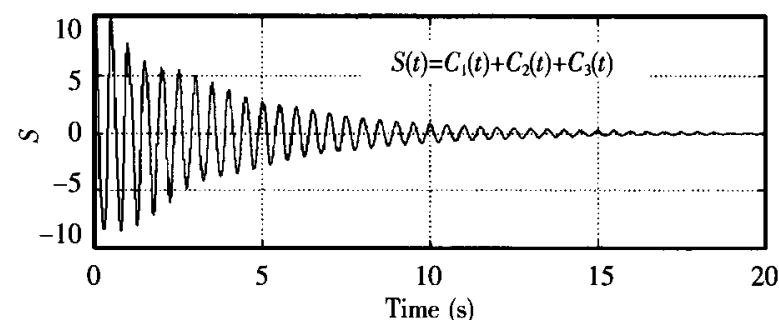

(a)

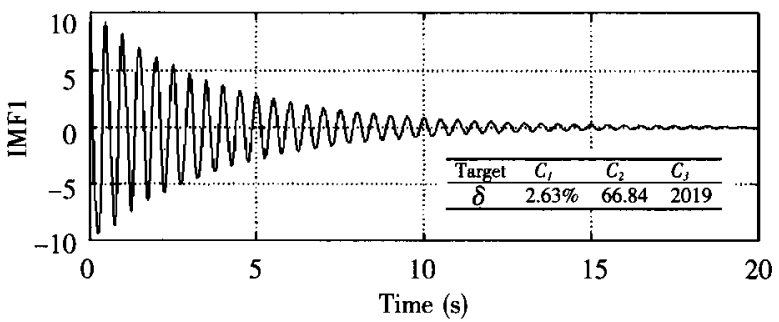

(c) extrema of the signal, which may lead to time scale misfit. In either case, the improvement of the efficiency of component decomposition is desirable.

This limitation of the EMD method is illustrated in Fig. 1, which is the EMD of a simulated signal imitating the free decay vibration. The simulated signal can be roughly accepted as an IMF, since it has equal number of local extrema and zero crossings and is almost symmetric; however it consists of three components, which the EMD method cannot distinguish. In this case, the EMD cannot identify the time scale of the riding waves, but ends up with a residual having a much longer time scale that bears no physical meaning. The deviations, $\delta$ ' $s$ in the figures are calculated by Eq. (27).

When more information of the system is to be obtained from the narrowband signals, both the resolution and the efficiency of the EMD method need to be improved. The technique proposed below can significantly improve the performance of the EMD method in this case.

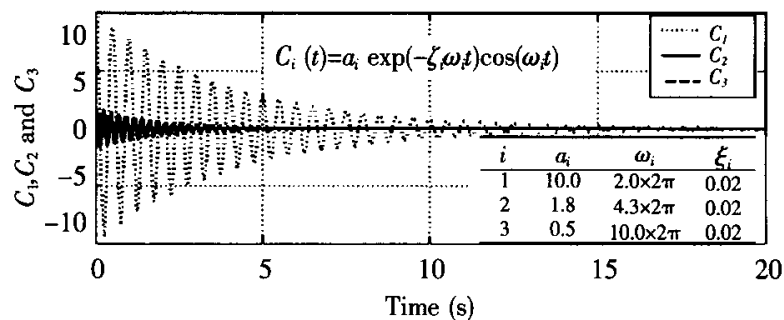

(b)

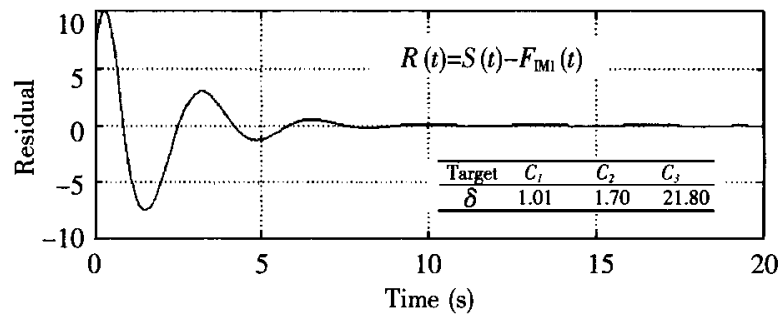

(d)

Fig. 1 A simulated 3-component signal and its EMD

\section{Proposed technique of component de- composition}

The proposed technique is based on the beating phenomena of waves, in which the envelope of the superposition of two waves will be oscillating at the frequency difference of the two waves. This phenomenon has been explored by Feldman to decompose a 2-component signal (Feldman, 1997). However, for real applications it has to be extended to signals with more than two components. Suppose that the signal $S(t)$ is the superposition of $n$ components :

$$
\begin{gathered}
S(t)=\sum_{i=1}^{n} C_{i}(t) \\
C_{i}(t)=A_{i}(t) \cos \left[\theta_{i}(t)\right], \theta_{i}(t)=t \omega_{i}(t)+\varphi_{i} \\
(i=1,2, \cdots, n)
\end{gathered}
$$

where $A_{i}(t)$ and $\omega_{i}(t)$ are positive functions gradually varying compared to $\cos \left[\theta_{i}(t)\right]$. Notice that $S(t)$ can be rewritten as: 


$$
\begin{aligned}
S(t) & =\left[\sum_{i=1}^{n} A_{i}(t) \cos \Delta \theta_{i 1}(t)\right] \cos \theta_{1}(t) \\
- & {\left[\sum_{i=1}^{n} A_{i}(t) \sin \Delta \theta_{i 1}(t)\right] \sin \theta_{1}(t) }
\end{aligned}
$$

in which and hereafter,

$\Delta \theta_{i j}(t)=\theta_{i}(t)-\theta_{j}(t)=t \Delta \omega_{i j}(t)+\Delta \varphi_{i j}$

$\Delta \omega_{i j}(t)=\omega_{i}(t)-\omega_{j}(t), \Delta \varphi_{i j}=\varphi_{i}-\varphi_{j}$

If $\sum_{i=1}^{n} A_{i}(t) \cos \Delta \theta_{i 1}(t)$ and $\sum_{i=1}^{n} A_{i}(t) \sin \Delta \theta_{i 1}(t)$ are varying not as fast as $\cos \theta_{1}(t)$ and $\sin \theta_{1}(t), \mathrm{Eq}$. (11) can be further represented as:

$$
\begin{gathered}
S(t)=a(t) \cos \left[\theta_{1}(t)+\alpha(t)\right], \\
a^{2}(t)=\sum_{i=1}^{n} A_{i}^{2}(t)+2 \sum_{i=1}^{n} \sum_{j=i+1}^{n} A_{i}(t) A_{j}(t) \cos \Delta \theta_{i j}(t) \\
\alpha(t)=\arctan \frac{\sum_{i=1}^{n} A_{i}(t) \sin \Delta \theta_{i 1}(t)}{\sum_{i=1}^{n} A_{i}(t) \cos \Delta \theta_{i 1}(t)}
\end{gathered}
$$

Therefore by Eq. (6), after the Hilbert transform, the squared instantaneous amplitude $a^{2}(t)$ of $S(t)$ will have two parts: the gradually varying trend $T(t)$ and the fluctuating part $F(t)$ oscillating with the frequency differences of any two components, where

$$
\begin{gathered}
T(t)=\sum_{i=1}^{n} A_{i}^{2}(t) \\
F(t)=2 \sum_{i=1}^{n} \sum_{j=i+1}^{n} A_{i}(t) A_{j}(t) \cos \Delta \theta_{i j}(t)
\end{gathered}
$$

Assume that the signal's energy is intense in its first several components, that is

$$
A_{1}(t) \gg A_{2}(t) \gg \cdots \gg A_{n}(t) .
$$

This assumption can be justified in many free decay vibration signals. As an example, the Fourier transform of a typical signal of traffic-induced bridge free vibration is illustrated in Fig. 2, where the energy carried by each component is indicated by the magnitudes of the corresponding peaks. Free decay signals can be obtained from impulse test of structures, or from ambient vibration measurement by the RandomDec technique (Ibrahim, 1977), or by selecting quasi-free decay segments from the signal, such as the segments when vehicles traveling off the bridge leave the bridge vibrating with no vehicles on it.

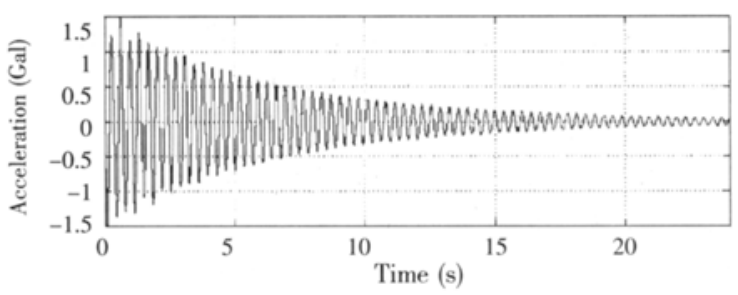

(a)

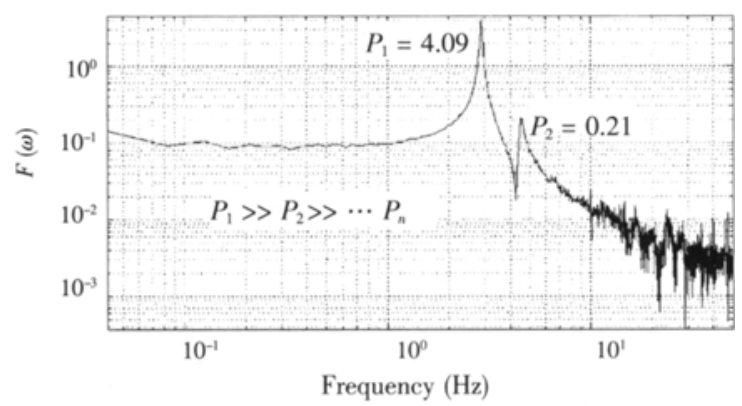

(b)

Fig. 2 A typical free decay signal from ambient vibration measurement of a bridge and its Fourier transform

With this assumption in Eq. (19), Eqs. (16), (17) and (18) can be approximately reduced to, respectively,

$$
\begin{gathered}
\alpha(t)=0 \\
T(t)=A_{1}^{2}(t) \\
F(t)=2 A_{1}(t) \sum_{j=2}^{n} A_{j}(t) \cos \Delta \theta_{j 1}(t)
\end{gathered}
$$

Therefore, a new component decomposition technique can be developed, consisting the following steps:

(1) Compute the Hilbert transform of the signal $S(t)$, and construct the analytic signal $Z(t)$ for $S(t)$ according to Eq. (2);

(2) Obtain the squared instantaneous amplitude $a^{2}(t)$ and the instantaneous phase $\theta(t)$ by Eqs. (3) and (4);

(3) Obtain the instantaneous frequency of the first component $\omega_{1}(t)$ by differentiating $\theta(t)$, for $\alpha(t)$ is approaching zero and $\theta(t)$ is approximately $\theta_{1}(t)$;

(4) Separate the gradually variant trend $T(t)$ and the fluctuation part $F(t)$ of $a^{2}(t)$, and find the instantaneous amplitude of the first component $A_{1}(t)$ by Eq. (21), therefore the first component can be fully reconstructed;

(5) Calculate the residual $R(t)$ which is defined by

$$
R(t)=\sum_{j=2}^{n} A_{j}(t) \cos \Delta \theta_{j 1}(t)=\frac{F(t)}{2 A_{1}(t)}
$$


and

(6) Treat the residual as the signal and repeat the procedure to obtain the next component, with the instantaneous amplitude $A_{2}(t)$ obtained in the same manner, but $\Delta \theta_{21}(t)$ adjusted to obtain the instantaneous phase and subsequently the frequency of the second component :

$$
\theta_{2}(t)=\Delta \theta_{21}(t)+\theta_{1}(t)
$$

The above procedure is repeated until the amplitude of the component is small enough to be ignored. In each round, the component with the largest energy is extracted and the order of the system is reduced by 1. The errors are accumulated to the components with less energy and thus less importance.

However, one issue remains to be solved: how to separate the trend $T(t)$ and the fluctuating part $F(t)$ in step (4). This study adopted the spline smoothing technique (Mathworks, 1999a) to estimate the trend, which gives the piecewise cubic function $T_{\mathrm{E}}(t)$ as an approximation of the trend $T(t)$ by minimizing

$$
D\left[T_{\mathrm{E}}\right]=\int_{\min (t)}^{\max (t)}\left[\frac{\mathrm{d}^{2} T_{\mathrm{E}}(\tau)}{\mathrm{d} t^{2}}\right]^{2} \mathrm{~d} \tau
$$

with a constrain

$$
\int_{\min (t)}^{\max (t)}\left[T_{\mathrm{E}}(\tau)-a^{2}(\tau)\right]^{2} \mathrm{~d} \tau \leq \varepsilon
$$

It may take several iterations to reach an appropriate $\varepsilon$, which will result in $F_{\mathrm{E}}(t)$ with almost symmetric upper and lower envelopes about $t$ axis. In the authors'practice, a low-pass filtered signal is used to estimate the value of $\varepsilon$, which is found satisfactory.

In this proposed component decomposition technique, the Hilbert transform unveils the time scale structure of the signal as a result of the beating phenomena of waves, the component extraction order is opposite to that of the EMD method, and the end effect of spline interpolation is somehow suppressed by employing a constrain condition for the full range of data. Therefore the proposed technique improves the EMD method in component decomposition for narrowband signals.

\section{Examples of the proposed method}

\subsection{Example 1}

To verify the proposed technique of component decomposition, the same simulated 3-component signal shown in Fig. 1 is decomposed into its three components by this technique. The $1^{\text {st }}, 2^{\text {nd }}$ and $3^{\text {rd }}$ rounds of component decomposition are illustrated in Figs 3,4 and 5 , respectively. The three components are reconstructed by their instantaneous amplitudes and instantaneous phases identified in this technique. The reconstructed components are compared to their theoretical counterparts in Fig. 6. Also notice here that the segments of the signal with ignorable small amplitude are cut off to avoid numerical difficulties. It is demonstrated that the proposed technique can identify the components of the signal of different time scales with satisfactory accuracy. As a result of this technique, the simulated signal is decomposed into its three mono-component components, which is verified in Fig. 7.

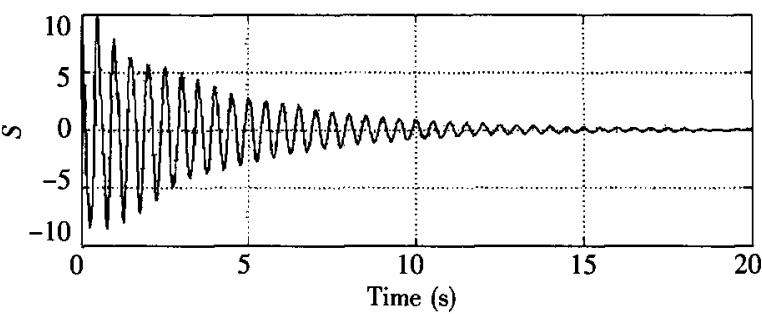

(a)

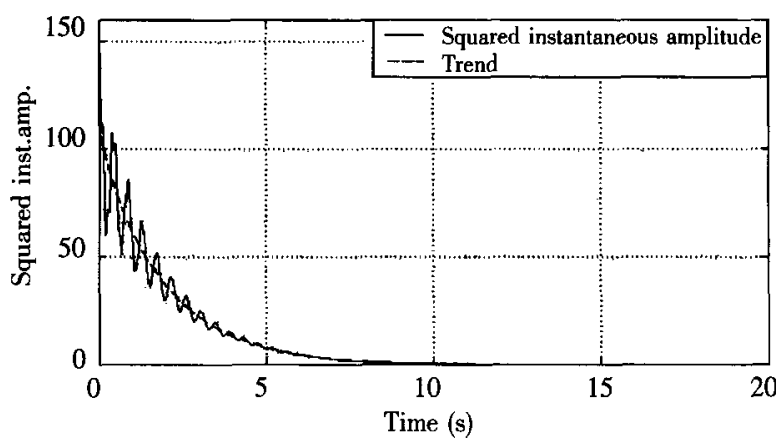

(b)

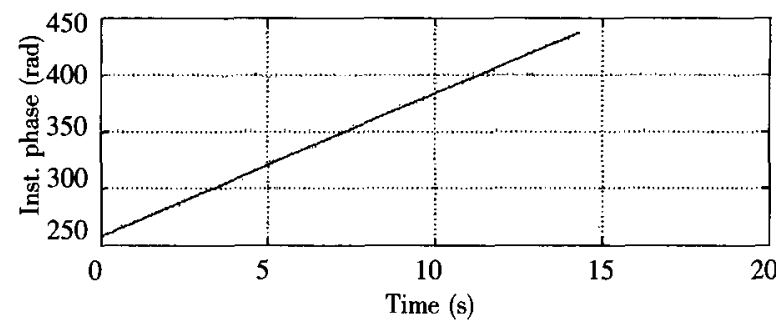

(c)

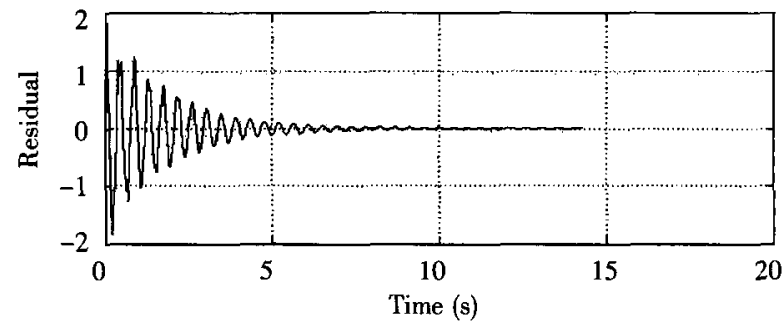

(d)

Fig. 3 First round component decomposition of the simulated signal in Fig. 1 


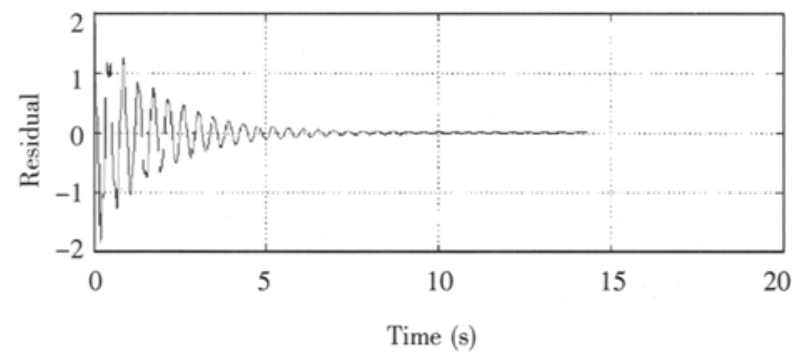

(a)

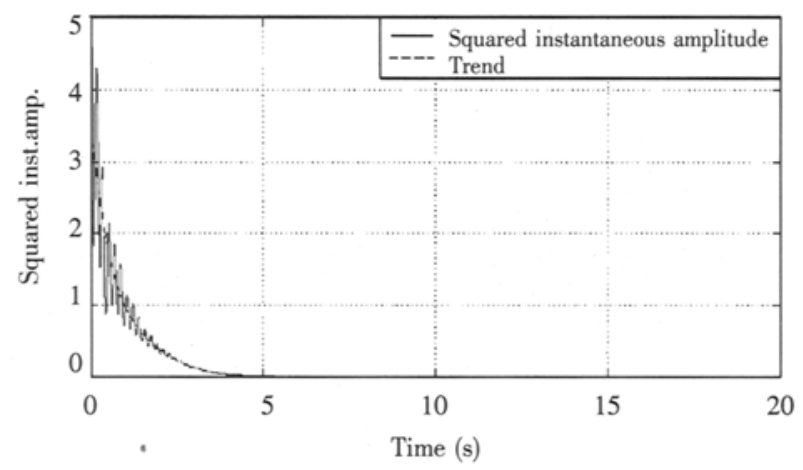

(b)

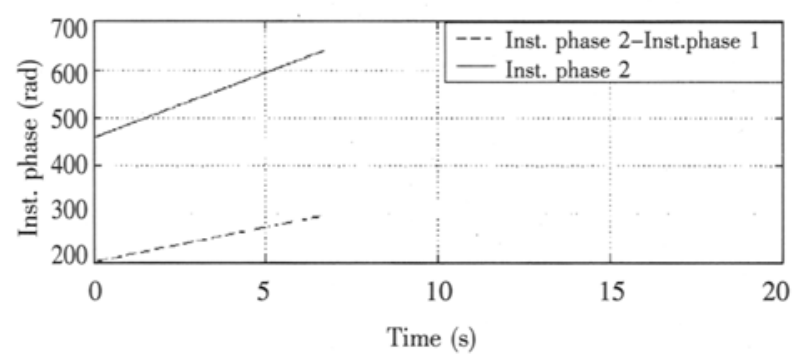

(c)

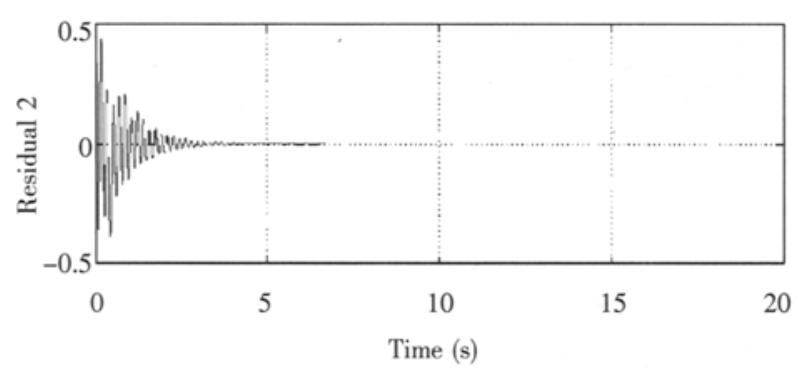

(d)

Fig. 4 Second round component decomposition of the simulated signal in Fig. 1

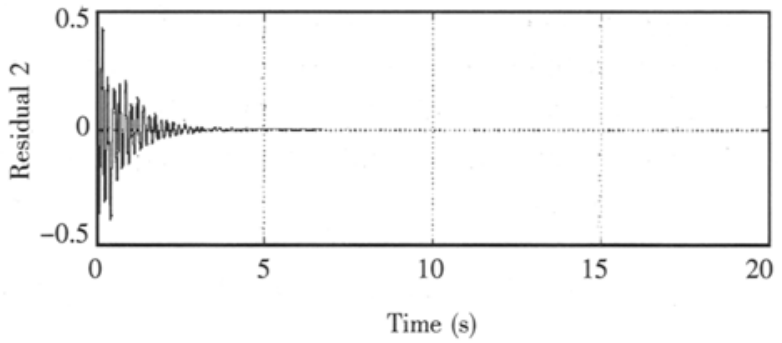

(a)

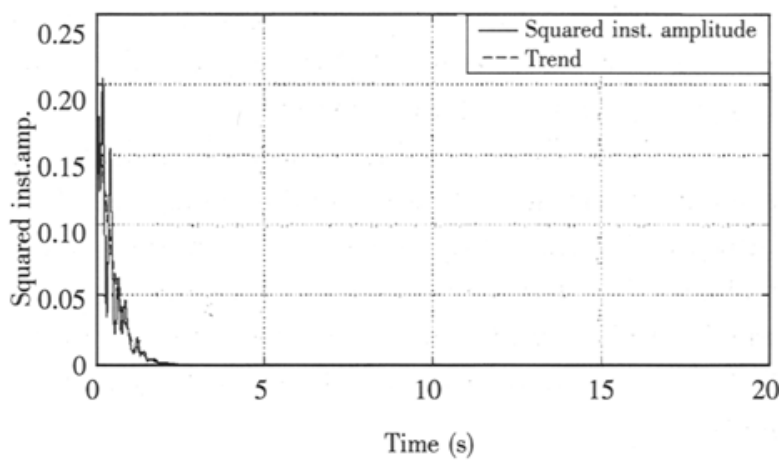

(b)

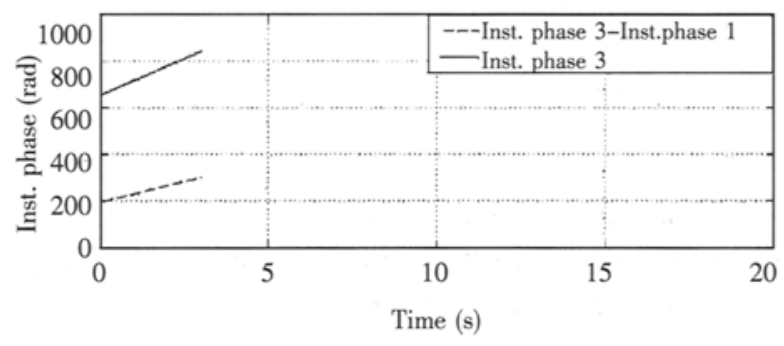

(c)

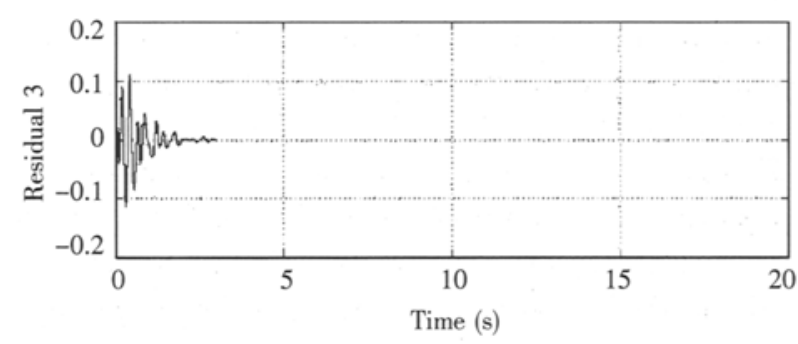

(d)

Fig. 5 Third round component decomposition of the simulated signal in Fig. 1 


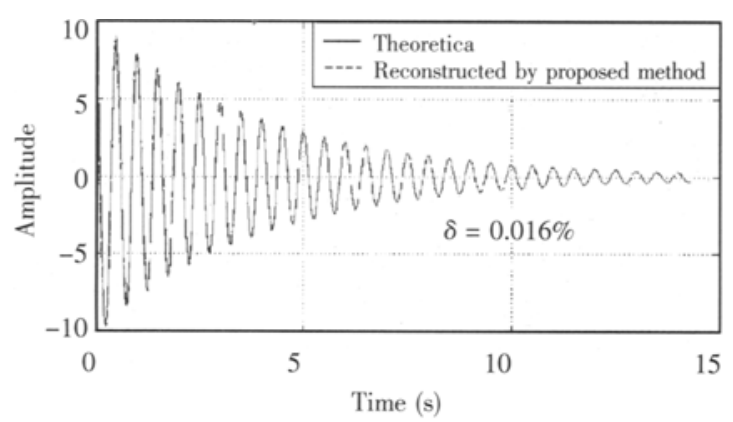

(a)Verification of the first component identified, $C_{1}$

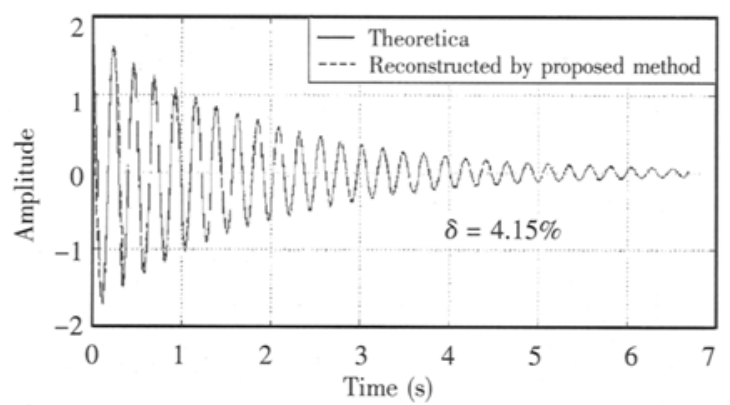

(b)Verification of the second component identified, $C_{2}$

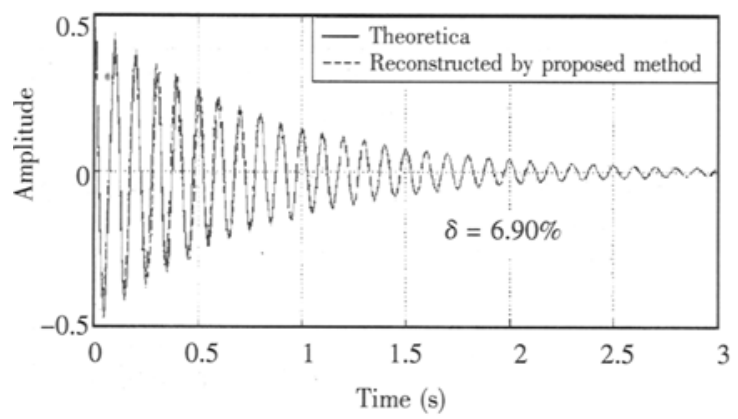

(c) Verification of the third component identified, $C_{3}$

Fig. 6 Verification of the identified components of the simulated signal in Fig. 1

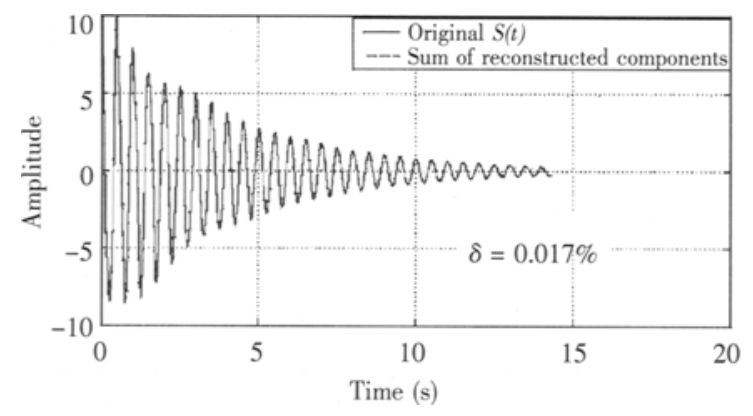

Fig. 7 Verification of the proposed technique by reconstructing the signal in Fig. 1 from identified components in Fig. 6

To evaluate the accuracy of the method, define the dimensionless deviation as

$$
\delta=\frac{\int_{\min (t)}^{\max (t)}\left[S_{R}(\tau)-S(\tau)\right]^{2} \mathrm{~d} \tau}{\int_{\min (t)}^{\max (t)} S^{2}(\tau) \mathrm{d} \tau}
$$

where $S_{R}(t)$ is the reconstructed signal and $S(t)$ is the target signal. The deviation $\delta$ of each of the components and that of the signal are also shown in each of the plots.

\subsection{Example 2}

To illustrate the capacity of the proposed technique for analyzing time-variant systems, a simple classical shear building model but with time varying stiffness is adopted as an example. The model has 2 DOFs as shown in Fig. 8. For simplicity, the properties of the model are normalized so that the initial stiffness of each story is unit, where $m_{1}=m_{2}=0.001 \mathrm{~kg}, k_{1}=1$ $\mathrm{N} / \mathrm{m}, k_{2}(t)=1-0.05 t \mathrm{~N} / \mathrm{m}$ where $t$ is the time variable in second, $c_{1}=c_{2}=0.0012 \mathrm{Ns} / \mathrm{m}$. Notice that the stiffness of the lower story is decreasing with a rate of $5 \%$ per second; at the end of the duration of the simulation, which is $12 \mathrm{~s}$, it is reduced to $40 \%$ of its initial value. The time histories of the displacement responses, $x_{1}(t)$ and $x_{2}(t)$, to the impulse excitation at $m_{1}$ are simulated in SIMULINK (Mathworks, $1999 \mathrm{~b}$ ) with a sampling frequency of $100 \mathrm{~Hz}$, and they are shown in Fig. 9.

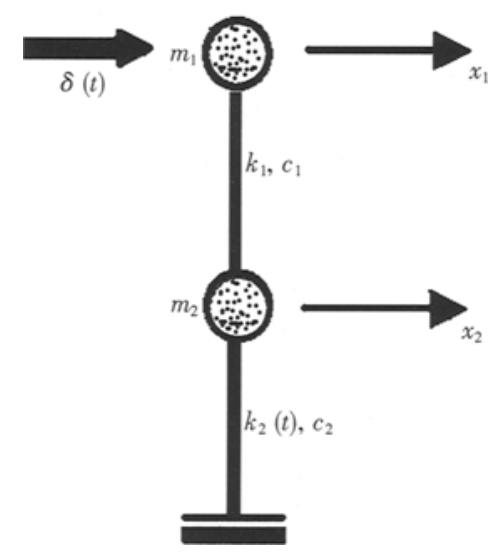

Fig. 8 A 2 - DOF shear building model with time-variant stiffiness

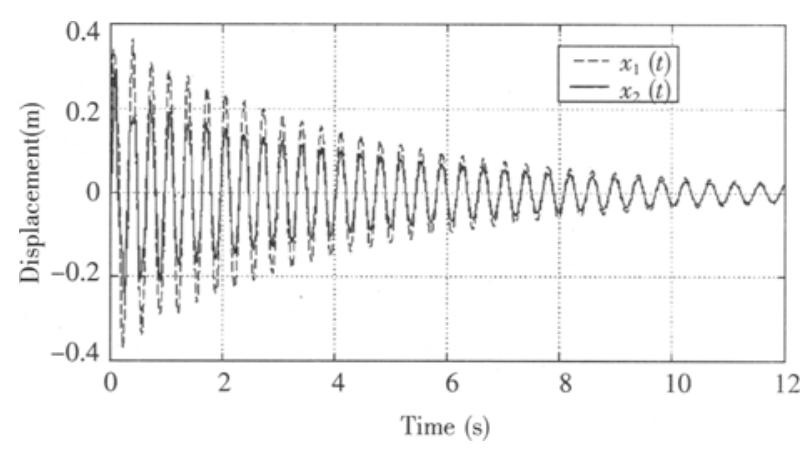

Fig. 9 Simulated displacement responses to the impulse excitation 
The equation of motion governing is

$$
\begin{gathered}
{\left[\begin{array}{cc}
m_{1} & 0 \\
0 & m_{2}
\end{array}\right]\left\{\begin{array}{l}
\ddot{x}_{1} \\
\ddot{x}_{2}
\end{array}\right\}+\left[\begin{array}{cc}
c_{1} & -c_{1} \\
-c_{1} & c_{1}+c_{2}
\end{array}\right]\left\{\begin{array}{l}
\dot{x}_{1} \\
\dot{x}_{2}
\end{array}\right\}+} \\
{\left[\begin{array}{cc}
k_{1} & -k_{1} \\
-k_{1} & k_{1}+k_{2}(t)
\end{array}\right]\left\{\begin{array}{l}
x_{1} \\
x_{2}
\end{array}\right\}=\left\{\begin{array}{l}
\delta(t) \\
0
\end{array}\right\}}
\end{gathered}
$$

where $\delta(t)$ is the unit impulse.

The Fourier transforms of $x_{1}(t)$ and $x_{2}(t)$ are show in Fig. 10, in which the Fourier transforms cannot reveal the time-variant nature of the system. The short time Fourier transform of one of the response signals $x_{2}(t)$ is also constructed and shown in Fig. 11, which, as can be seen, has unsatisfactory resolution to represent the time variance of the system. This can be understood by looking at the fact that to achieve a resolution of $0.1 \mathrm{~Hz}$, the window width should be at least 1000 points when sampling at $100 \mathrm{~Hz}$, but the total length of the $12 \mathrm{~s}$ data is only 1200 points, which is merely enough for only one window, not allowing it to slide along the time axis without overlapping. Examining the signal $x_{2}(t)$, it should have two components (the system has 2 DOFs), which again the EMD cannot distinguish. Applying the proposed technique, the signal is decomposed into its two components as shown in Fig. 12. The Hilbert transforms of both components give the instantaneous frequency for each of the components as shown in Fig. 13. The instantaneous frequencies of both components decrease vs. time, revealing the time-variant property of the system, despite the end effect of Hilbert transform and the fluctuation due to the spline approximation (which are exaggerated by differentiating the instantaneous phase to obtain the instantaneous frequency).

\subsection{Example 3}

The proposed technique is also applied to the free

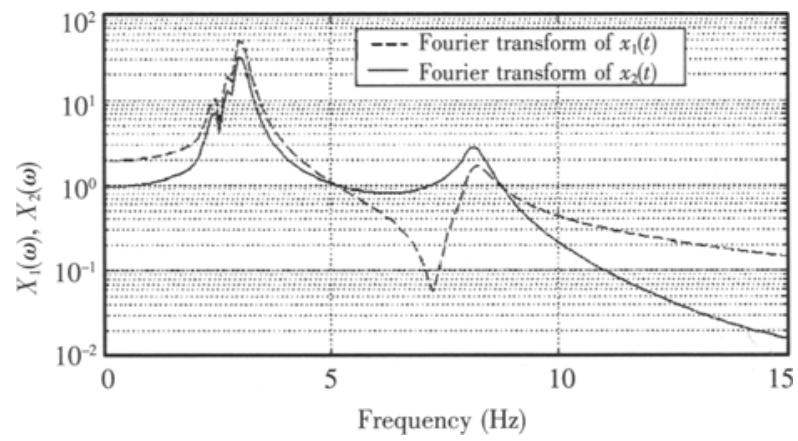

Fig. 10 The Fourier transforms of $x_{1}(t)$ and $x_{2}(t)$.

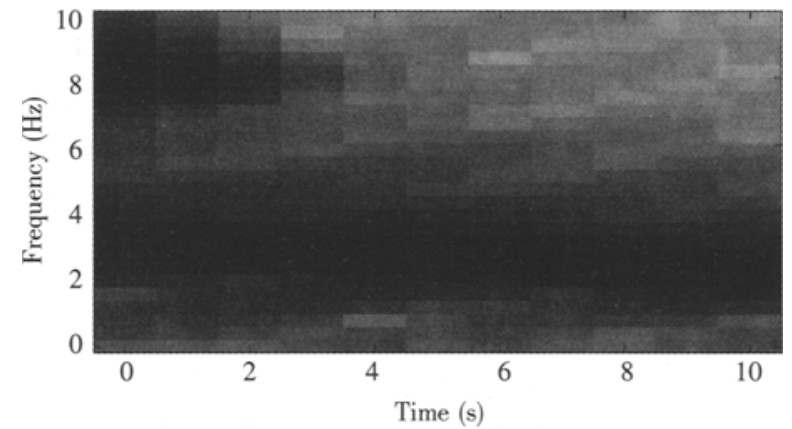

Fig. 11 The short time Fourier transform of $x_{2}(t)$ (number of points of FFT:256, width of window : 200, number of points overlapping: 100 )

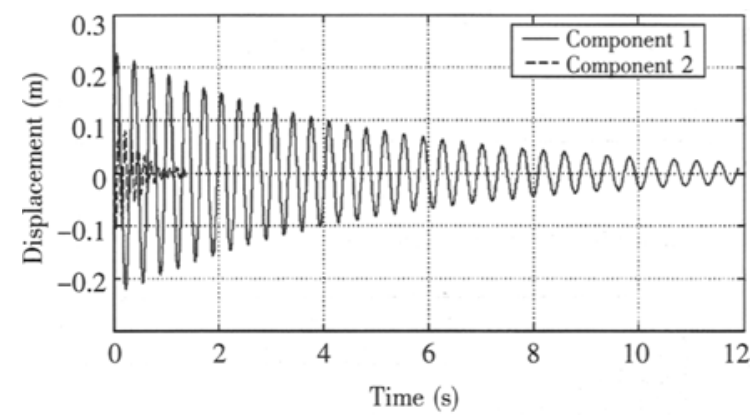

Fig. 12 Component decomposition of $x_{2}(t)$ by the proposed technique

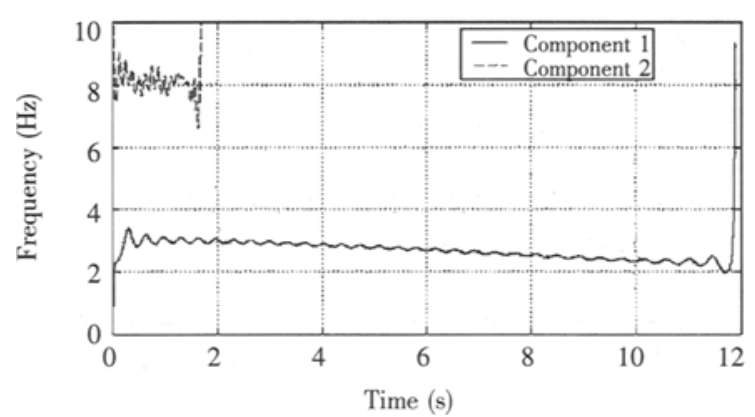

Fig. 13 The instantaneous frequencies of both components of $x_{2}(t)$

decay signals obtained from the traffic-induced vibration measurement of an instrumented highway concrete bridges monitored by the authors (Feng and Kim, 2001 ). The signal shown in Fig. 2 is taken as an example. The first and second components are extracted in the first two rounds of the component decomposition. The decomposition demonstrates sufficient accuracy in Fig. 14. Therefore the Hilbert spectrum (Huang et al. , 1998; 1999) of the free decay signal can be calculated and presented in Fig. 15 with Gaussian smoothing. 


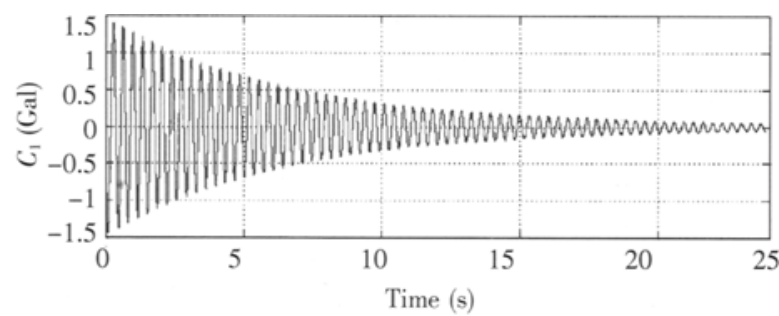

(a) Component $1 \quad C_{1}(t)$

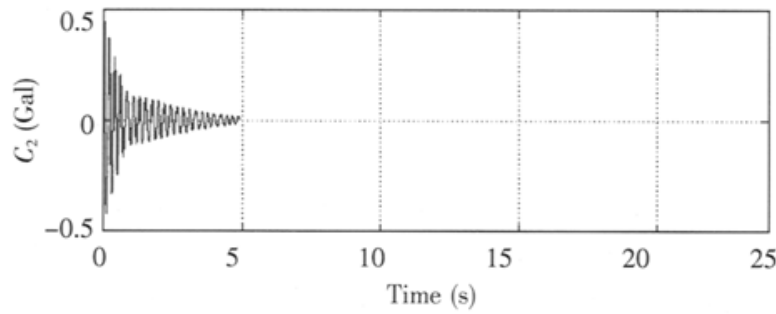

(b) Component $2 C_{2}(t)$

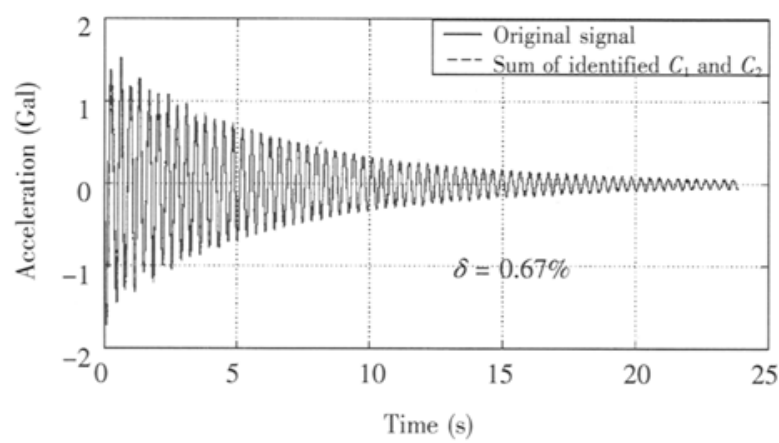

(c) Comparison of the signal and the sum of reconstructed components

Fig. 14 The component decomposition of the measured signal in Fig. 2

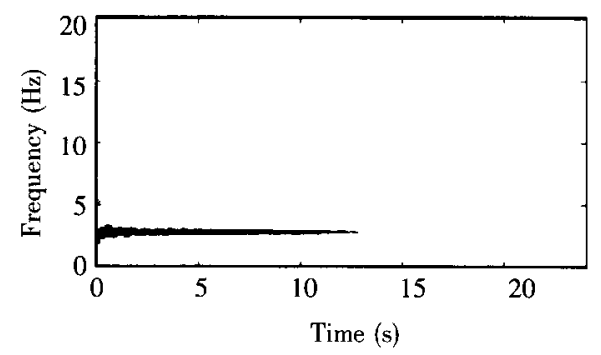

Fig. 15 The Hilbert spectrum of the measured signal in Fig. 2( with 9 by 9 Gaussian smoothing. $s t d=1.2$ )

\section{Conclusion}

The time-frequency domain analysis based on the Hilbert transform has the capacity to localize the timevariant characteristics of the structural system. The signal has to be decomposed into its components beforehand to admit a well-behaved Hilbert transform. The idea of component decomposition is first implemented by the EMD, which, however, has its limitation in distinguishing components in narrowband signals often obtained in real vibration measurement. A new technique for decomposing component based on waves' beating phenomena is proposed to improve the EMD, in which the time scale structure of the signal is unveiled by the Hilbert transform as a result of the beating phenomenon, the order of component extraction is opposite to that of the EMD and the end effect is confined. The proposed technique is verified by performing the component decomposition of a simulated signal and a free decay signal measured in a real bridge structure. The capability of the proposed technique in capturing the time-variant characteristics of dynamic systems is demonstrated with a simulated 2DOF shear-building model with time-variant stiffness. The proposed technique can achieve satisfactory accuracy in reconstructing the components imbedded in the narrowband signals, which are commonly found in structures'free decay responses. However, the identification of the instantaneous frequency and damping characteristics involves differentiation, which is vulnerable to noise or numerical error. This will be studied in the future.

The potential application of the proposed technique in structural health monitoring remains to be explored.

\section{References}

Feldman M (1994a), “Non-Linear System Vibration Analysis Using Hilbert Transform-I. Free Vibration Analysis Method 'FREEVIB'," Mechanical Systems and Signal Processing, 8(2) : 119-127.

Feldman M (1994b), “Non-Linear System Vibration Analysis Using Hilbert Transform-II. Forced Vibration Analysis Method 'FORCEVIB'," Mechanical Systems and Signal Processing, 8(3) : 309-318.

Feldman M ( 1997 ), “Non-linear Free Vibration Identification via the Hilbert Transform," Journal of Sound and Vibration, 208(3) : 475-489.

Feng MQ and Kim D (2001), “Long-Term Structural Performance Monitoring of Two Highway Bridges, Phase I: Instrumentation," Report to the California Department of Transportation RTA-59A0155.

Gröchenig K (2001), Foundations of Time-Frequency Analysis, Birkh user, Boston.

Hahn SL (1996), Hilbert Transform in Signal Processing, Artech House, Boston.

Huang NE, Shen Z and Long SR (1999), “A New View of Nonlinear Water Waves: the Hilbert Spectrum," Annu. Rev. Fluid Mech., 1999, 31: 417457.

Huang NE, Shen Z, Long SR, Wu MC, Shih HH, 
Zheng Q, Yen N, Tung CC and Liu HH (1998),

"The Empirical Mode Decomposition and the Hilbert Spectrum for Nonlinear and Non-stationary Time Series Analysis," Proc. R. Soc. Lond. A 454, pp. 903995.

Ibrahim SR (1977), "Random decrement technique for modal identification of structures," Journal of Spacecraft and Rocket, AIAA,14(11).

Mallat S (1999), A Wavelet Tour of Signal Processing, Second Edition, Academic Press, San Diego.

Mathworks (1999a), Spline Toolbox User's Guide, The MathWorks Inc, Natick, MA, USA.

Mathworks (1999b), Using SIMULINK, The MathWorks Inc, Natick, MA, USA.

Pan J, Yan X, Zheng Q, Liu TW, and Klemas VV ( 2003 ), “Interpretation of Scatterometer Ocean
Surface Wind Vector EOFs over the Northwestern Pacific," Remote Sensing of Environment, 84(1) :53-68 Salvino LW (2000), “Empirical Mode Analysis of Structural Response and Damping," Proceedings SPIE the International Society for Optical Engineering, 1 pp. 503-509.

Yang JN and Lei Y (2000), "System Identification of Linear Structures Using Hilbert Transform and Empirical Mode Decomposition," Proceedings SPIE the International Society for Optical Engineering, 1 pp. 213219.

Zhang RR and Ma S (2000), “HHT Analysis of Earthquake Motion Recordings and its Implications to Simulation of Ground Motion," International Conference on Monte Carlo Simulation, Monte Carlo, June $18-21$, pp. $483-490$. 